\title{
Divalent Metal- and High Mobility Group N Protein-Dependent Nucleosome Stability and Conformation
}

\author{
Michelle S. Ong, Dileep Vasudevan, and Curt A. Davey \\ Division of Structural and Computational Biology, School of Biological Sciences, Nanyang Technological University, \\ 60 Nanyang Drive, Singapore 637551 \\ Correspondence should be addressed to Curt A. Davey, davey@ntu.edu.sg
}

Received 14 July 2010; Accepted 29 September 2010

Academic Editor: Emery H. Bresnick

Copyright ( $\odot 2010$ Michelle S. Ong et al. This is an open access article distributed under the Creative Commons Attribution License, which permits unrestricted use, distribution, and reproduction in any medium, provided the original work is properly cited.

\begin{abstract}
High mobility group N proteins (HMGNs) bind specifically to the nucleosome core and act as chromatin unfolding and activating factors. Using an all-Xenopus system, we found that HMGN1 and HMGN2 binding to nucleosomes results in distinct iondependent conformation and stability. HMGN2 association with nucleosome core particle or nucleosomal array in the presence of divalent metal triggers a reversible transition to a species with much reduced electrophoretic mobility, consistent with a less compact state of the nucleosome. Residues outside of the nucleosome binding domain are required for the activity, which is also displayed by an HMGN1 truncation product lacking part of the regulatory domain. In addition, thermal denaturation assays show that the presence of $1 \mathrm{mM} \mathrm{Mg}^{2+} i$ or $\mathrm{Ca}^{2+}$ gives a reduction in nucleosome core terminus stability, which is further substantially diminished by the binding of HMGN2 or truncated HMGN1. Our findings emphasize the importance of divalent metals in nucleosome dynamics and suggest that the differential biological activities of HMGNs in chromatin activation may involve different conformational alterations and modulation of nucleosome core stability.
\end{abstract}

\section{Introduction}

Histone proteins package eukaryotic DNA into chromatin, a multilevel array in which nucleosomes comprise the basic unit [1]. The nucleosome consists of a core, $\sim 147$ base pairs (bp) wrapped around a histone octamer in 1.67 left-handed turns [2], in addition to a variable length of linker DNA. By influencing DNA site exposure and factor association, nucleosome positioning provides a platform for regulation, such as gene-specific control of transcription [3]. In conjunction with a variety of histone variant substitutions, posttranslational modifications, and chromatin remodeling activities, nucleosome organization and dynamics allow for site selectivity in genomic transactions well beyond DNA primary structure.

An additional regulatory feature of chromatin entails compaction status and nucleosome structure. Many nuclear activities require open chromatin states and at least transient alteration of nucleosomes $[4,5]$. As the most abundant nonhistone proteins in the nucleus, high mobility group factors assist in these processes by modifying chromatin "architecture" [6]. Within this protein family, HMGNs are unique in having specific affinity for the nucleosome core, wherein they act to facilitate processes including DNA repair, replication, and transcription by unfolding chromatin $[7,8]$.

HMGN variants modulate distinct histone modifications [9], and they display cell cycle-dependent binding to chromatin [10] and differential expression during development [11]. Under physiological conditions, HMGN1 and HMGN2 bind cooperatively to the nucleosome core to form species containing two molecules of either one or the other variant (not mixed pairs) $[10,12]$. Specificity and affinity are conferred by an $\sim 30$ amino acid N-terminal motif - the nucleosome-binding domain (NBD) $[13,14]$. In contrast to the highly positive-charged NBD, the C-terminus contains a very acidic region, encompassing the regulatory domain $(\mathrm{RD})$, which is required for high-affinity binding to chromatin [14], transcription stimulation, and disruption of linker histone H1-mediated chromatin compaction $[15,16]$.

In spite of extensive biochemical and functional analysis of the HMGNs, their chromatin-modifying activities are intricate, and mechanisms of action have remained elusive. 
In this work, we have composed a reconstituted Xenopus laevis system to investigate the nature of structural and thermodynamic alterations arising from HMGN-nucleosome interactions. Our findings hold significance for understanding the architectural changes in chromatin elicited by this family of nuclear factors.

\section{Materials and Methods}

2.1. HMGN Production. Xenopus laevis HMGN1 and HMGN2 expression constructs were generated by inserting codon-optimized genes into the NdeI and BamHI sites of pET-3a vector (EZBiolab Inc., Westfield, USA). Proteins were overexpressed in E. coli and purified using a similar approach as applied previously for recombinant HMGNs [17]. The supernatant of the cell lysate, obtained by homogenization in a buffer of $50 \mathrm{mM}$ Tris- $\mathrm{HCl}$ ( $\mathrm{pH} 7.5$ ), $0.5 \mathrm{M} \mathrm{NaCl}, 1 \mathrm{mM}$ EDTA, $1 \mathrm{mM} \beta$-mercaptoethanol, and $1 \mathrm{mM}$ phenylmethylsulphonyl fluoride (PMSF), was subjected to size-exclusion chromatography using a 26/60 Sephacryl S-200 column (GE Healthcare, Uppsala, Sweden) pre-equilibrated with a buffer of $20 \mathrm{mM}$ Tris- $\mathrm{HCl}$ ( $\mathrm{pH} 7.5$ ), $0.2 \mathrm{M} \mathrm{NaCl}, 1 \mathrm{mM}$ EDTA, and $1 \mathrm{mM}$ PMSF. Further chromatographic purification was carried out using a Mono S or Resource S cation-exchange column (GE Healthcare, Uppsala, Sweden) equilibrated with a buffer of $20 \mathrm{mM}$ Tris- $\mathrm{HCl}$ (pH 7.5), $0.1 \mathrm{M} \mathrm{NaCl}$, and $1 \mathrm{mM}$ EDTA. HMGN eluted over a gradient of 0.2 to $0.4 \mathrm{M} \mathrm{NaCl}$ (20 mM Tris-HCl (pH 7.5), 1 mM EDTA). Purified HMGN1 (11.4 kD), HMGN2 $(9.4 \mathrm{kD})$, and HMGN1t $(8.9 \mathrm{kD})$ were subjected to $\mathrm{N}$-terminal sequencing and mass spectrometry analysis to establish composition.

2.2. Nucleosomal Materials. Nucleosome core particle (NCP) was assembled with Xenopus laevis histones as described previously [18]. NCP147 and NCP146b are composed, respectively, of $147 \mathrm{bp}$ and 146 bp derivatives of human $\alpha$ satellite DNA [19]. Off-centered NCP146b was generated as described before [20]. NCP-601 was produced from a $145 \mathrm{bp}$ fragment, atcagaatcccggtgccgaggccgctcaattggtcgtagacagctctagcaccgcttaaacgcacgtacgcgctgtcccccgcgttttaaccgccaaggggattactccctagtctccaggcacgtgtcagatatatacatcgat, corresponding to the strong positioning "601" nucleosome core element [21].

4-nucleosome array and 12-nucleosome array (gifts from T. Richmond, ETH-Zurich), composed, respectively, of $167 \mathrm{bp}$ and $177 \mathrm{bp}$ repeats with the 601 core element [21], were produced using a modified version of the original protocol $[22,23]$. Trace amounts of residual plasmid vector fragments arising from the EcoRV digest for 12nucleosome array or exogenously added 145 bp DNA [24] for 4-nucleosome array served as an excess histone octamer sink during reconstitution to prevent oversaturation of the array DNA. Contaminating NCP and small nucleosomal assemblies were eliminated by differentially precipitating array with the addition of 2.5 to $4 \mathrm{mM} \mathrm{MgCl}$. Histone octamer saturation of array was confirmed via ScaI restriction digestion analysis, whereby cleavage occurs at the linker DNA midpoint between nucleosome sites (see Supplementary
Figure 1 in Supplementary Material available online at doi: 10.4061/2010/143890, which includes 5 figures).

2.3. Electrophoretic Mobility Shift Assays. Electrophoretic mobility shift assays (EMSAs) were carried out with three different running buffers; approximate physiological ionic strength buffer corresponded to 1X TBE $(89 \mathrm{mM}$ Tris$\mathrm{HCl}, 89 \mathrm{mM}$ boric acid, $1.0 \mathrm{mM}$ EDTA, and $\mathrm{pH} 8.3$ ), and low ionic strength buffer was, with two exceptions, $0.25 \mathrm{X}$ TBE. For the 12-array samples in the upper panels of Figure 2(a) and Figure 3(c), the buffer was 0.25X TAE (10 mM Tris- $\mathrm{HCl}, 10 \mathrm{mM}$ acetic acid, $0.25 \mathrm{mM}$ EDTA, and $\mathrm{pH}$ 8.3). Electrophoresis under divalent metal conditions was conducted using a $1 \mathrm{X}$ TB buffer $(89 \mathrm{mM}$ Tris- $\mathrm{HCl}, 89 \mathrm{mM}$ boric acid, and $\mathrm{pH} 8.3$ ) with the addition of $1 \mathrm{mM} \mathrm{MgCl} 2$ or $\mathrm{CaCl}_{2}$. Gels were loaded with aliquots having nucleosome core site concentrations of $0.5 \mu \mathrm{M}$ for both NCP and 12-array samples.

Preassembled NCP or array was incubated with HMGN or NBD peptide in respective $0.25 \mathrm{X}$ or $1 \mathrm{X}$ running buffer for approximately $30 \mathrm{~min}$ at room temperature prior to electrophoresis at $4^{\circ} \mathrm{C}$. For coassembly trials, HMGN was introduced either at the onset of salt dialysis-based histoneDNA reconstitution or at a $\mathrm{KCl}$ concentration of $0.4 \mathrm{M}$, corresponding to the "midpoint." NCP was analyzed with $6 \%$ polyacrylamide gels, whereas analysis of array was conducted using $1 \%$ agarose $1.3 \%$ polyacrylamide composite gels.

2.4. Thermal Denaturation Assays. HMGN was added at desired stoichiometry to $0.75 \mu \mathrm{M}$ DNA or NCP in $1 \mathrm{X}$ TB or $1 \mathrm{X} \mathrm{TB}+1 \mathrm{mM} \mathrm{MgCl} 2$ buffer, and samples were allowed to incubate at room temperature for at least 10 minutes before taking measurements using a Varian Cary 300 Bio UV/Vis spectrophotometer equipped with a temperature controller. Data collection entailed monitoring UV absorbance at $260 \mathrm{~nm}$ wavelength from $\sim 20^{\circ}$ to $\sim 95^{\circ} \mathrm{C}$ over $1^{\circ}$ intervals. First derivative of absorbance versus temperature values $(\Delta H / \Delta T)$ were calculated with the instrument software using standard settings. Initial $\Delta H / \Delta T$ versus temperature profiles were normalized by adjusting with respect to a $\Delta H / \Delta T$ maximum setting of 1.0 (yielding $\Delta H / \Delta T^{*}$ values in Figure 3).

\section{Results}

3.1. HMGN Binding Can Give Rise to a Distinct SpeciesS2. In the production of recombinant Xenopus HMGN1 and HMGN2, we also obtained a C-terminal truncation product, HMGN1t, lacking 25 amino acids from the RD (Figure 1). The three proteins bind cooperatively to form 2:1 HMGN : nucleosome core particle (NCP) species (S1) at near physiological ionic strength (see Figure 1(a)). The relative electrophoretic mobility of S1 for HMGN1 compared to HMGN2 changes with buffer conditions, whereby HMGN1-S1 migrates slowest at low ionic strength. At near physiological ionic strength, the relative migration becomes roughly equivalent, and with the addition of $1 \mathrm{mM} \mathrm{Mg}^{2+}$, HMGN2-S1 is slowest. Therefore, binding of two molecules 
$\begin{array}{lll}-1- & -2- & -3-\end{array}$

n N1t N1 N2 N1t N1 N2 N1t N1 N2
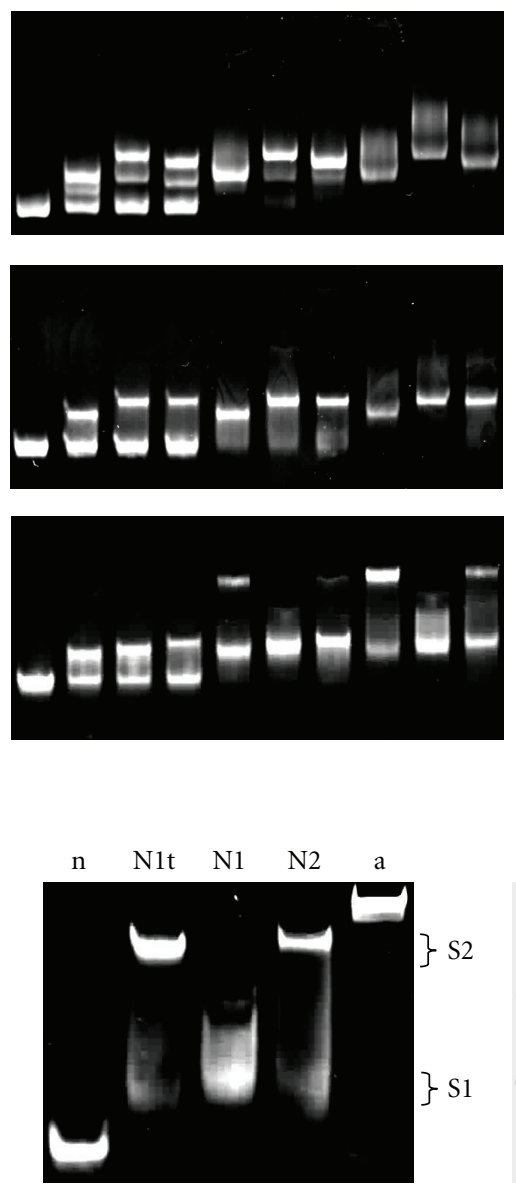

(b) $\begin{array}{llll}-1- & -2- & -3-\end{array}$

n N1t N1 N2 N1t N1 N2 N1t N1 N2
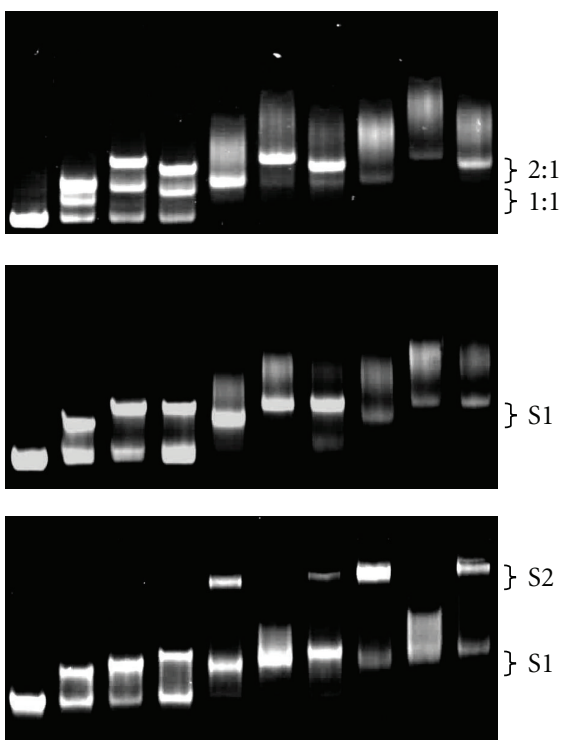

(a)

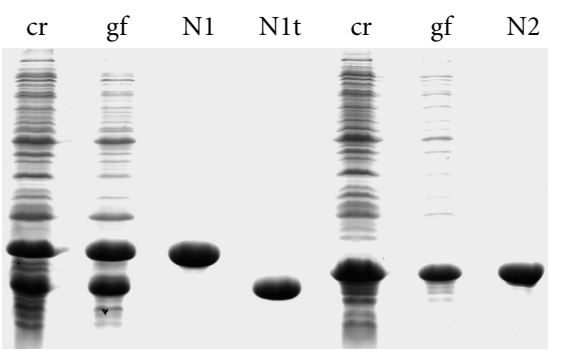

(c)

HMGN1

HMGN1t $\nabla$

PKRKQVNADVADAKDEPKRRSARLSSKPTPAKTEPKPKKEKAPVKEKPEEKEKKVPAKGKKGAKGKQTEEANKEEANEDQPSENGETKSDEAPASDGGDKESKSE HMGN2

PKRKADGDSKAEKAKAKDEPQRRSARLSAKPAPPKPEAKPKKAAAPPKKADKAPKGKKGKADSGKDSSNAAENGEAKSDQAQKAETGDTK

(d)

FIGURE 1: HMGN binding to the nucleosome core is ion dependent and capable of eliciting a transition. Gel labels indicate HMGN identity (N1t, HMGN1t; N1, HMGN1; N2, HMGN2; n, NCP alone; a, nucleosome array alone), and numbers designate HMGN : nucleosome molar stoichiometry. (a) EMSAs of HMGN binding to two distinct NCP constructs, NCP-601 (left) and NCP147 (right), under three different buffer conditions: low ionic strength (top), near physiological ionic strength (middle), and near physiological ionic strength with $1 \mathrm{mM} \mathrm{Mg}{ }^{2+}$ (bottom). Under noncooperative binding conditions (top), $1: 1$ as well as $2: 1 \mathrm{HMGN}$ : nucleosome species are observed at low stoichiometry [12]. At higher ionic strength (middle), 2:1 species (S1) are the only specific complexes that occur. In the presence of divalent metal, a distinct, slow-migrating species (S2) is also observed for HMGN1t and HMGN2 binding. (b) EMSA showing migration rate for S1 and S2 of NCP-601 (HMGN : nucleosome $=4: 1$ ) relative to NCP and a 4-nucleosome array. (c) SDS-polyacrylamide gel analysis of HMGN purification products (see Methods). Samples from HMGN1 (left) and HMGN2 (right) overexpression are shown for crude extract (cr) and after gel filtration (gf) and ion-exchange chromatography (N1, N1t, and N2). (d) Xenopus HMGN primary structures. Functional domains are the NBD (blue) and RD (red). Nuclear localization elements are shown in green.

of HMGN1 or HMGN2 to the NCP results in different iondependent conformations.

In the presence of $\mathrm{Mg}^{2+}$ and at two and above protein to NCP molar ratio, a distinct species (S2) is observed for HMGN2 or HMGN1t binding, which displays dramatically reduced electrophoretic mobility (see Figures 1(a) and 1(b)).
This S1-S2 transition has no apparent DNA sequence dependence since both NCP constructs (NCP147 and NCP-601), composed of unrelated sequences, display nearly identical behavior. Furthermore, $\mathrm{Mg}^{2+}$-dependent $\mathrm{S} 2$ formation is also observed for HMGN1t or HMGN2 binding to a 12nucleosome array (12-array; Figure 2). Similar to NCP, 

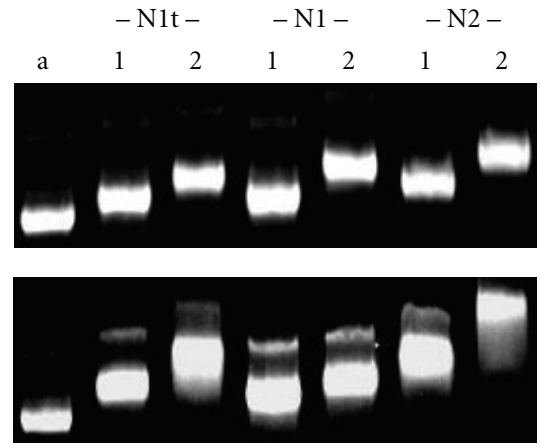

(a)

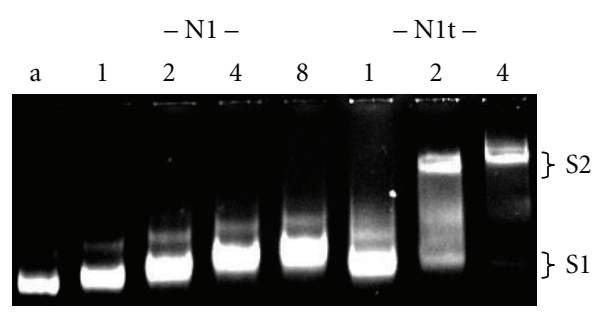

(b)

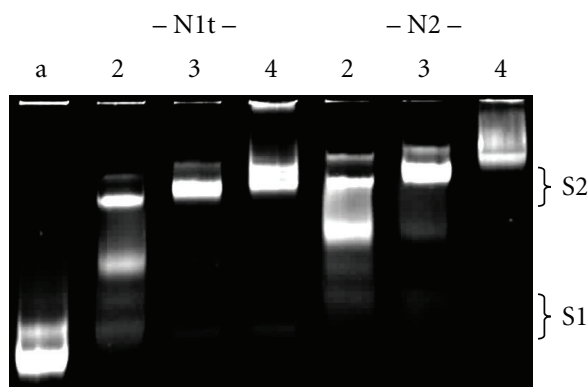

(c)

FIGURE 2: HMGN association with nucleosomal array is capable of inducing the S2 transition. Gel labels indicate HMGN identity, (N1t, HMGN1t; N1, HMGN1; N2, HMGN2; a, 12-array alone) and numbers designate HMGN : nucleosome molar stoichiometry. (a) HMGN binding to 12-array at low (top) and near physiological ionic strength (bottom). ((b), (c)) HMGN binding to 12-array at near physiological ionic strength with $1 \mathrm{mM} \mathrm{Mg}^{2+}$.

HMGN : nucleosome ratios of 2:1 and above can trigger the S2 transition for the 12-array.

3.2. Elements Required for S2 Formation. In order to determine the basic components required for S2 formation, we investigated whether the phenomenon is $\mathrm{Mg}^{2+}$ specific. However, $\mathrm{Ca}^{2+}$ is equally effective at eliciting the transition, which therefore displays a divalent metal dependency (Figure 3(a)). Also, considering that the truncated form of HMGN1 is capable of causing S1-S2 transition, we tested whether the NBD alone has such activity. Yet, 32 amino acid peptides corresponding to the NBD of either HMGN1 or HMGN2 (see Figure 1(d)) are unable to evoke S2, even at very high stoichiometry (Figures 3(b) and 3(c)). Therefore, the residues necessary for producing the transition reside outside the NBD of HMGN1 and HMGN2. Moreover,

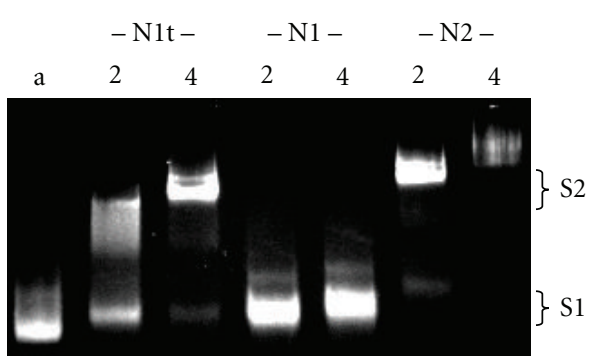

(a)

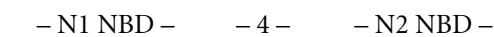

$\begin{array}{llllllllll}\mathrm{n} & 4 & 7 & 10 & \mathrm{~N} 1 \mathrm{t} & \mathrm{N} 1 & \mathrm{~N} 2 & 4 & 7 & 10\end{array}$

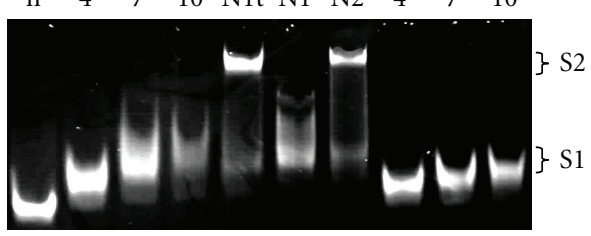

(b)

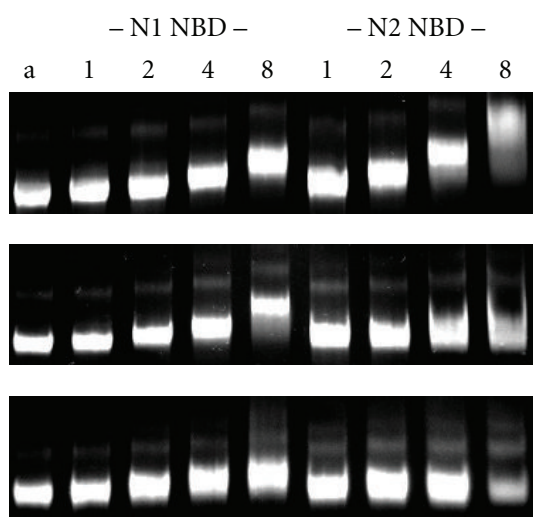

(c)

FIgURE 3: S2 formation is divalent metal dependent and requires HMGN elements outside of the NBD. EMSA gel labels indicate HMGN identity (N1t, HMGN1t; N1, HMGN1; N2, HMGN2; a, 12-array alone; n, NCP alone), and numbers designate HMGN : nucleosome molar stoichiometry. (a) HMGN binding to 12 -array at near physiological ionic strength with $1 \mathrm{mM} \mathrm{Ca}^{2+}$. (b) Binding of HMGN proteins and NBD peptides to NCP-601 at near physiological ionic strength with $1 \mathrm{mM} \mathrm{Mg}^{2+}$. (c) Binding of HMGN NBD peptides to 12-array at low ionic strength (top), near physiological ionic strength (middle), and near physiological ionic strength with $1 \mathrm{mM} \mathrm{Mg}^{2+}$ (bottom).

although the HMGN1 NBD peptide is able to bind to the 12array at near physiological ionic strength in the presence of $\mathrm{Mg}^{2+}$, association of the HMGN2 NBD peptide under these chromatin compacting conditions [22] is not observed (see Figure 3(c)). It is important to note that the HMGN2 NBD peptide does bind readily to NCP under identical conditions (see Figure 3(b)).

3.3. S2 Entails a Conservative Reversible Transition. The electrophoretic mobility shift assays (EMSAs) reveal analogous behavior for both NCPs and the 12-array, and the extreme reduction in migration rate of $\mathrm{S} 2$ relative to $\mathrm{S} 1$ 


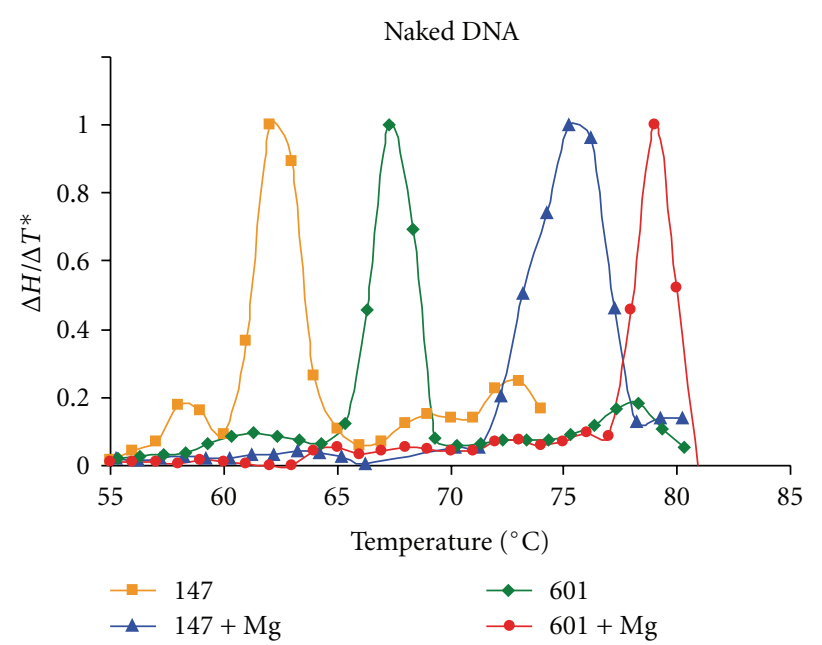

(a)

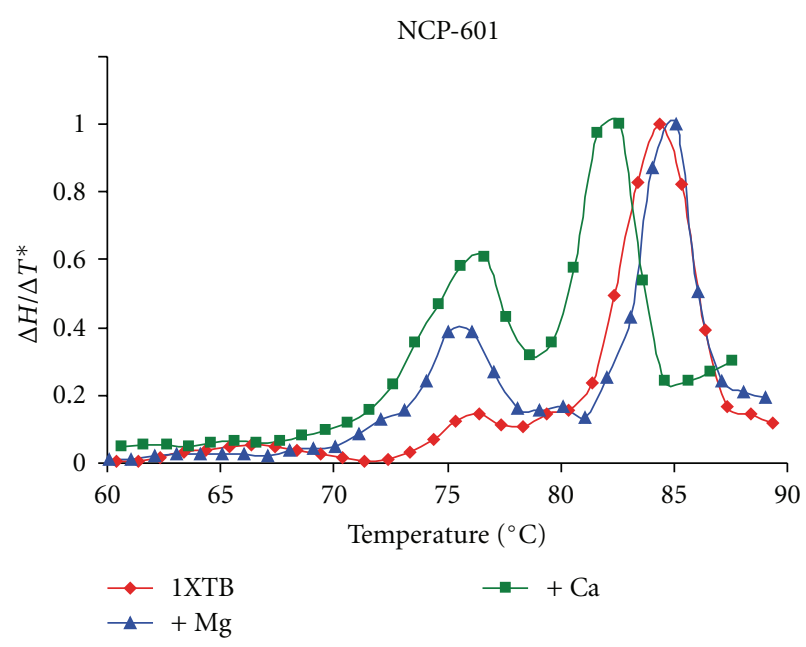

(b)

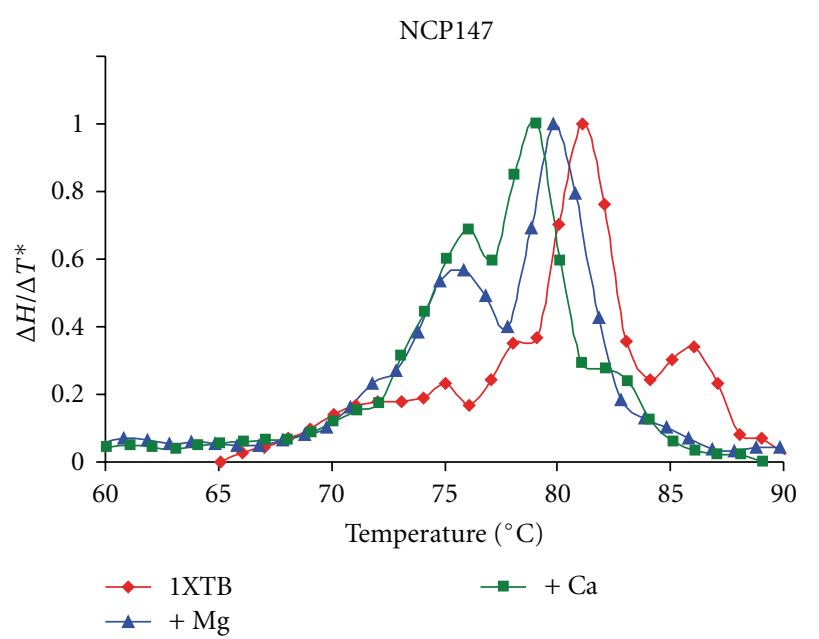

(c)

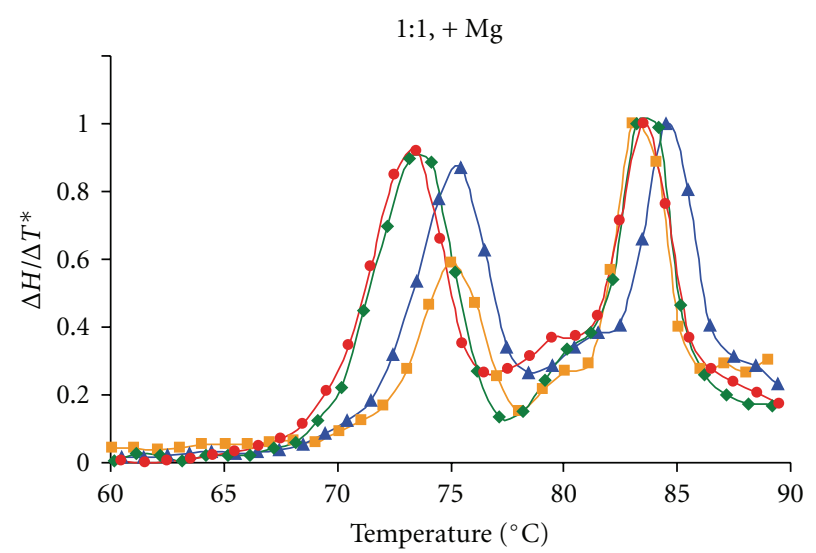

$\begin{array}{ll}-\mathrm{NCP} & \rightarrow \mathrm{N} 1 \mathrm{t} \\ -\mathrm{N} 1 & \rightarrow \mathrm{N} 2\end{array}$

(e)

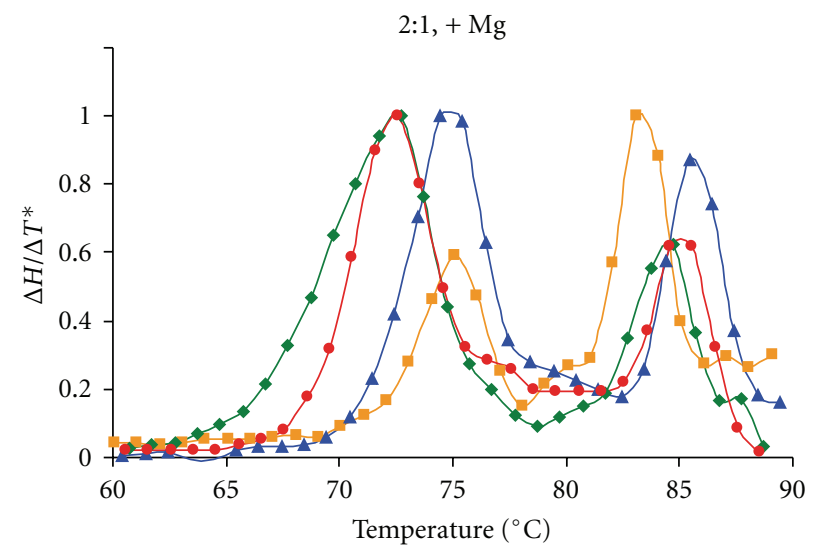

(f)

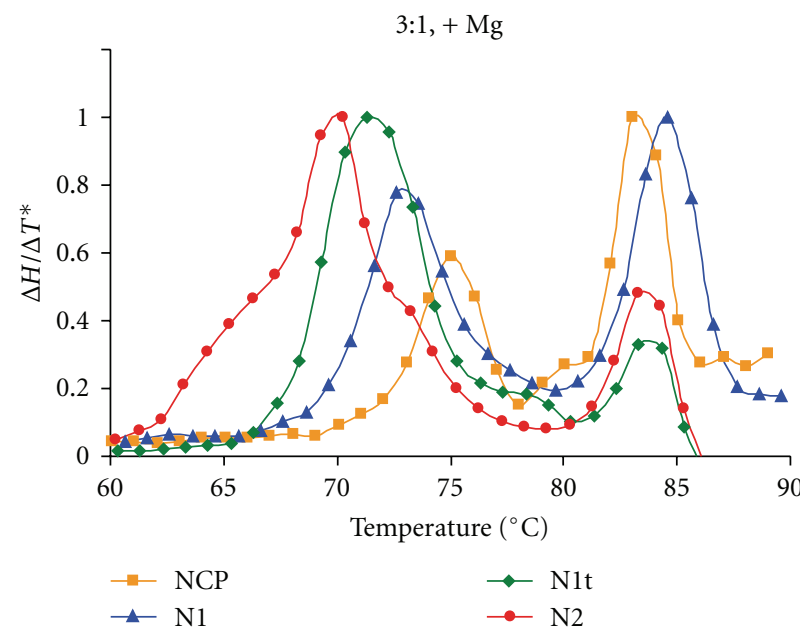

(g)

Figure 4: Continued. 


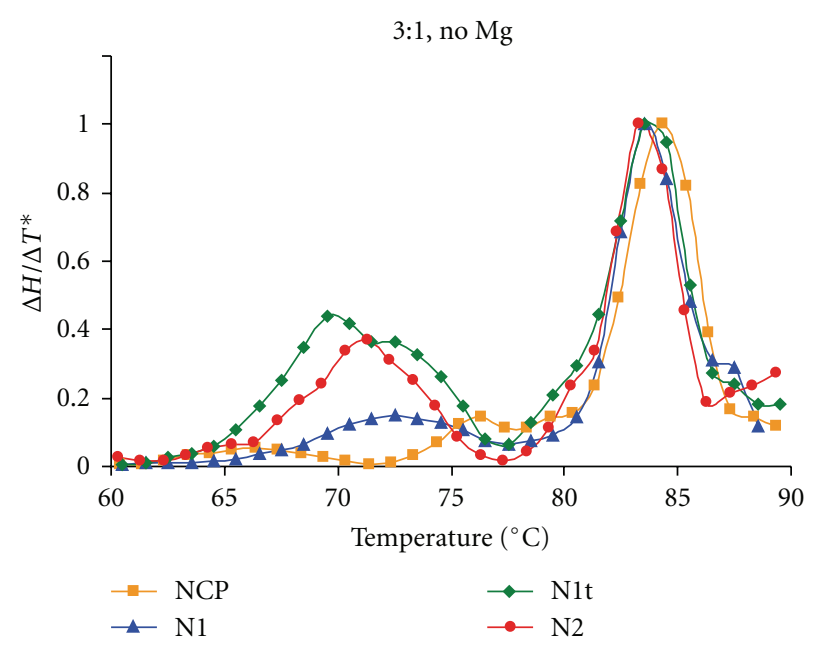

(d)

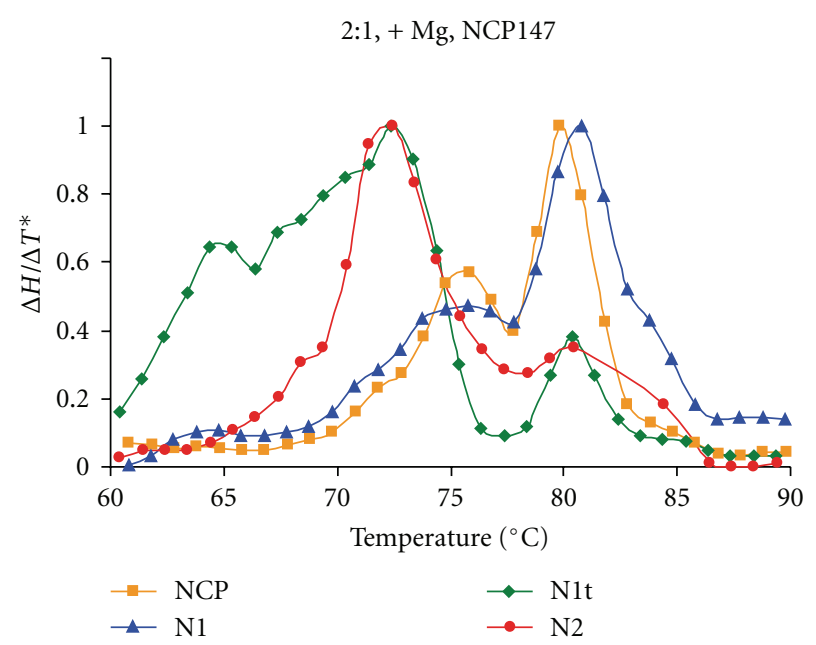

(h)

FIGURE 4: Thermal denaturation assays reveal influence of divalent metal and HMGNs on nucleosome stability. ((a)-(h)) Sample buffer was near physiological ionic strength without divalent metal ((a)-(d); $1 \mathrm{XTB}$, default) or with $1 \mathrm{mM} \mathrm{Mg}^{2+}$ or Ca $\mathrm{Ca}^{2+}((\mathrm{a})-(\mathrm{c}),(\mathrm{e})-(\mathrm{h})$; +Mg, $+\mathrm{Ca})$. Assays were conducted on naked NCP DNA fragments alone (a), NCP ((b), (c)), NCP-601 with 3:1 HMGN: NCP stoichiometry in the absence of divalent metal (d), NCP-601 with $1: 1$ (e), $2: 1$ (f), or 3:1 (g) HMGN : NCP stoichiometry and NCP147 with $2: 1$ HMGN : NCP stoichiometry (h). ((d)-(h)) "NCP" corresponds to an HMGN-free control sample.

is consistent with a pronounced conformational change of the nucleosome core to a less compact state. The nature of this transition suggests that it could involve irreversible nucleosome disassembly or modification. However, we find that the S1-S2 conversion can be completely reversed by either removing $\mathrm{Mg}^{2+}$ or reducing the HMGN : nucleosome stoichiometry (Supplementary Figure 2).

Although the S1-S2 transition is readily reversible, structural rearrangements in the nucleosome may be influenced by the presence of cofactors during chromatin assembly. We therefore tested whether introduction of HMGN at the beginning or midpoint of the nucleosome reconstitution process, as opposed to protein addition after assembly, affects the outcome (Supplementary Figures 3 and 4). Differences could arise, for instance, through competition between HMGN and H2A-H2B dimer for the same DNA-binding sites. However, NCP or 12-array coassembled with HMGN behaves in an apparently identical fashion as preassembled material, with the former also capable of S1 and S2 formation.

\subsection{Divalent Metal Presence and HMGN Binding Reduce} Nucleosome Core Terminus Stability. In order to delineate the influence of buffer condition and HMGN binding, we conducted thermal denaturation experiments to assess nucleosome stability (Figure 4) [25-28]. This assay is based on the hyperchromic effect of increased DNA UV absorbance arising from base unstacking. In this way, the unwinding of the double helix can be monitored, yielding a sigmoidal melting profile, for which the first derivative is equivalent to change in enthalpy with respect to temperature $(\Delta H / \Delta T)$. The sigmoidal inflexion points, or maximal $\Delta H / \Delta T$ values, correspond to melting temperatures $\left(T_{m}\right)$ associated with one or more transitions. Moreover, the relative areas in $\Delta H / \Delta T$ plots associated with multiple transitions are proportional to the relative extents of double helix unwinding.

Thermal denaturation of the nucleosome is generally associated with two transitions [25-28]. The minor component occurs first and corresponds to unwinding of the DNA termini. This is followed by the major transition at elevated temperature, in which the remaining double helix melts. As observed previously for samples in monovalent cation buffer, the magnitude of the minor transition is much smaller relative to the major transition for NCP thermally denatured in near physiological ionic strength buffer (see Figures 4(b) and $4(\mathrm{c})$ ). However, with the addition of $1 \mathrm{mM} \mathrm{Mg}^{2+}$ or $\mathrm{Ca}^{2+}$, the minor transition becomes much more pronounced, indicative of a substantially greater extent of DNA unwinding arising from divalent metal-mediated destabilization of the nucleosome core termini. Moreover, the $T_{m}$ of the major transition can be lowered from the presence of divalent metal-in particular for $\mathrm{Ca}^{2+}$. The overall destabilization of the NCP from the presence of divalent metal is particularly striking in comparison with the naked DNA fragments, which display the opposite behavior as a consequence of double helix stabilization by $\mathrm{Mg}^{2+}$ and $\mathrm{Ca}^{2+}$ (see Figure 4(a)). As a result, under the present divalent metal conditions, the $T_{m}$ for the initial minor NCP transition is similar to the $T_{m}$ for the respective naked DNA fragment. This indicates that, from the modulating influence of divalent cations, histone octamer association may provide only weak stabilization of the terminal DNA arms in the nucleosome.

In conjunction with the divalent metal conditions, the presence of saturating stoichiometry of either HMGN2 or HMGN1t shows a profound further reduction in stability of the nucleosome termini relative to NCP alone 


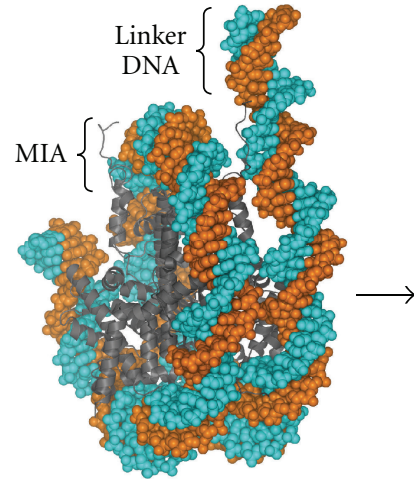

Off-centered $(\mathrm{O})$

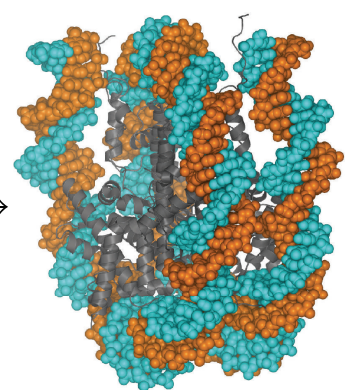

Centered (C) (a)

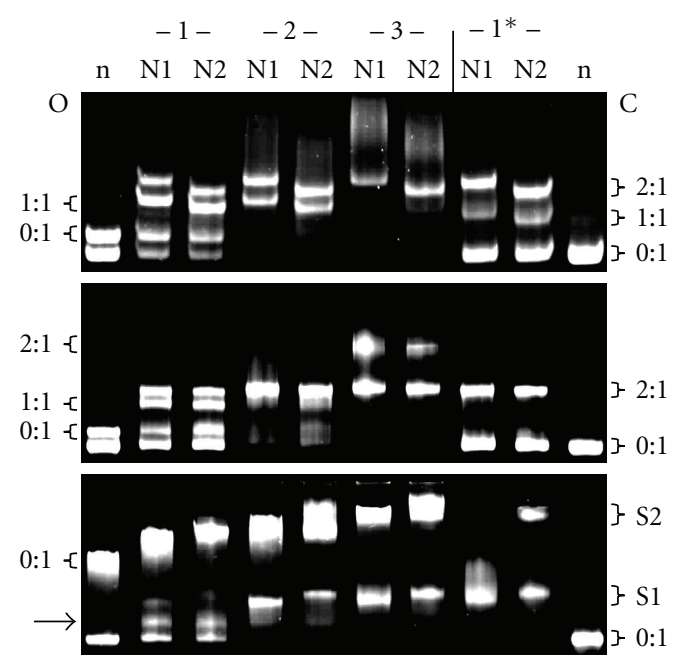

(b)

FIGURE 5: Off-centered NCP undergoes a transition in the presence of divalent metal and associates with HMGNs to form an S2like state. (a) Crystal structure-based [19] models for off-centered and centered NCP. DNA strands are colored orange and cyan and histone proteins are grey. Off-centered NCP is missing an interaction between one DNA terminus and the $\mathrm{N}$-terminal tail of H3 (MIA). (b) EMSAs of HMGN binding to centered (C; right) versus off-centered (O; left) NCP146b. Gel labels indicate HMGN identity (N1t, HMGN1t; N1, HMGN1; N2, HMGN2; n, NCP alone), and numbers designate HMGN:nucleosome molar stoichiometry. Off-centered samples (left 7 lanes) also contain a fraction of faster migrating centered NCP. Gels were run under three different buffer conditions: low ionic strength (top), near physiological ionic strength (middle), and near physiological ionic strength with $1 \mathrm{mM} \mathrm{Mg}^{2+}$ (bottom). HMGN : nucleosome molar stoichiometry $(*)$ for centered samples (right 3 lanes) is $1: 1$ (top, middle) or $3: 1$ (bottom). Assignments of $1: 1$ and $2: 1 \mathrm{HMGN}$ : off-centered NCP species are inferred by comparison with those for centered NCP (see text for details). The additional band observed at low HMGN stoichiometry with $\mathrm{Mg}^{2+}$ (arrow) may coincide with a minor fraction of off-centered NCP in a compact state associated with one molecule of HMGN. (see Figures 4(e)-4(h)). Moreover, what is initially the minor transition at lower temperature under HMGN-free conditions becomes the main transition, encompassing the majority of the double helix. In addition, the $T_{m}$ for this early transition is significantly reduced relative to NCP alone. Thus, both the ease and extent of unwinding the nucleosome core termini is increased dramatically with HMGN2 or HMGN1t association.

Although a slight destabilization of the termini is apparent from saturating amounts of HMGN2 or HMGN1t in near physiological ionic strength monovalent cation buffer, the major effect is clearly contingent on the presence of divalent metal (see Figure 4(d)). In contrast to the divalent metal-dependent influence of HMGN2 or HMGN1t association, for HMGN1 binding, one sees a much diminished effect on reducing nucleosome termini stability (see Figures 4(d)-4(h)).

3.5. Off-Centered Nucleosome Core Particle Undergoes a Transition in the Presence of Divalent Metal. Reconstitution of NCP typically yields off-centered and centered forms, which differ in positioning of the histone octamer on the DNA by $10 \mathrm{bp}$ (Figure 5). Compared to the thermodynamically favored centered NCP, the off-centered form lacks a histone H3 N-terminal tail-DNA interaction at the recessed end and contains an additional section of linker DNA on the opposing half. In order to illuminate potential mechanistic features for HMGN-induced decompaction of the nucleosome, we investigated protein binding to off-centered NCP.

Under low ionic strength conditions, a distinct specific species for HMGN binding to off-centered NCP is observed, which has electrophoretic mobility intermittent between the $1: 1$ and $2: 1$ species seen for purely centered NCP (see Figure 5(b)). Moreover, under near physiological ionic strength conditions, where cooperative binding to centered NCP occurs, two additional specific species are observed for off-centered NCP. The migration rates of the new offcentered species relative to those for centered NCP and their prevalence at either only low or high HMGN stoichiometry suggests that they coincide with $1: 1$ and 2:1 assemblies. The existence of a $1: 1$ off-centered complex at physiological ionic strength would in turn imply that cooperative HMGN binding to the nucleosome core requires intact elements at the termini.

In the presence of $\mathrm{Mg}^{2+}$, off-centered NCP displays a dramatic reduction in electrophoretic mobility relative to the centered form, consistent with a substantial decrease in compactness of the nucleosome core (see Figure 5(b) and Supplementary Figure 5). Furthermore, in addition to HMGN2 and HMGN1t, HMGN1 can associate with this off-centered species to generate an assembly with migration properties very similar to S2. This suggests that disruption of histone-DNA interactions at one terminus may render the nucleosome core susceptible to transition to a decompacted conformation, to which HMGNs can stably associate.

\section{Discussion}

HMGN1 and HMGN2 have both been shown to enhance transcription from and promote nuclease digestion of 
minichromosomes assembled in nuclear/ovum extracts, but the effect has largely been observed only if HMGN is present during, as opposed to being introduced after, chromatin assembly [7, 16, 29, 30]. Here, we have constructed a bottomup system in order to uncover fundamental distinctions between HMGN variants. We do not observe any significant differences arising from whether HMGN is present from the start or midpoint of nucleosome assembly, as compared to protein addition subsequent to reconstitution. In conjunction with the observation that a significant fraction of HMGN in the nucleus is associated with metastable multiprotein complexes [31], this indicates that there are likely multiple factors, such as histone tail modifications [13], which can regulate or influence HMGN activity in vivo.

Although HMGN1 and HMGN2 are known to modulate distinct activities $[9,11]$ and localize to separate regions in the nucleus [32], differences in chromatin structural attributes associated with the two variant types are not known. With the trials of divalent metal-containing conditions notwithstanding, our results do not reveal any pronounced conformational or stability differences of HMGNnucleosome assemblies between the two variants. On the other hand, association of HMGN2 in the presence of $\mathrm{Mg}^{2+}$ or $\mathrm{Ca}^{2+}$ - the ubiquitous divalent cations in the nucleus [33]-leads to a substantial reduction in stability of the nucleosome core DNA termini and appears to trigger a conformational switch in the nucleosome to a less compact state. However, in contrast to HMGN2, we do not observe a pronounced stability or structural transition elicited through HMGN1 association, which is nonetheless consistent with a previous HMGN1-nucleosome array investigation [34]. One possibility is that HMGN1 requires additional cofactors or enzymatic modification. Although residues $\mathrm{N}$-terminal to the NBD may play a role, the activity we observe is likely conferred by elements within or in the vicinity of the $\mathrm{RD}$, since it is also displayed by our C-terminal truncation species, HMGN1t, but not by the NBD peptides. In fact, HMGN1t is similar to the HMGN3b variant, which also lacks a substantial portion of the RD compared to HMGN1 [35]. In this regard, the acidic 10 amino acid region at the C-terminus of HMGN1t has similar character compared to an element within the HMGN2 RD situated at roughly identical distance in primary structure from the NBD (see Figure $1(\mathrm{~d})$ ). Thus, variations in the HMGN C-terminus arising from differences in expression or posttranslational modification could allow fine regulation of nucleosome stability and structural transitions.

HMGN1 and HMGN2 have been found to localize to separate clusters of nucleosomes in vivo [32], and this differential clustering in the nucleus is promoted a priori by the exclusive nucleosome binding behavior of these two variants. The distinct conformational and thermodynamic properties we observe for HMGN1 versus HMGN2 assemblies support a previously proposed allosteric mechanism underlying the absence of mixed variant pairs bound to an individual nucleosome [12]. In low ionic strength buffer, the electrophoretic migration rate for S1 displays a typical proportionality to the molecular weight of the HMGNNCP assembly. Under more physiological ionic conditions, however, the HMGN2-S1 migrates equally or more slowly with respect to HMGN1-S1, in spite of the greater mass of the latter. This suggests a conformational change to a somewhat less compact state upon HMGN2 association with the nucleosome under cooperative binding conditions. In addition, under approximate physiological ionic conditions with $\mathrm{Mg}^{2+}$, the HMGN1 NBD peptide can associate with NCP or 12-array, whereas binding of the HMGN2 NBD peptide to NCP, but not 12-array, is observed. Considering that the isolated HMGN2 NBD also displays homopaired association suggests that the binding of this NBD alone elicits a conformational change as well [12], which is suppressed by nucleosome compaction. Thus, overall it appears that binding of one HMGN2 induces a conformational change in the nucleosome, which facilitates association of a second HMGN2 while preventing that of HMGN1.

Although previous work has shown that association of HMGN1 and HMGN2 increases the stability of the nucleosome core, these thermal denaturation studies were conducted at low ionic strength with monovalent cation buffers [28]. In contrast to the inhibitory effect of $\mathrm{Mg}^{2+}$ and $\mathrm{Ca}^{2+}$ on dissociation of the double helix [36], we find a pronounced stability reduction for the nucleosome core in the presence of only low concentrations of these divalent cations. Although there is a general decrease in $T_{m}$ for the final high-temperature transition, the primary influence relates to destabilization of the DNA ends at the nucleosome core termini. We recently completed a crystallographic study characterizing in detail counterion binding in the nucleosome core and found that divalent metal hydrates and the histone N-terminal tails can compete with each other for association with the same sites in the DNA minor groove [37]. Therefore, considering that the terminal histone-DNA interactions are the weakest and most dynamic $[19,38-40]$, the main divalent metal-mediated stability reduction apparently arises through binding competition, such that the nucleosomal DNA arms are more frequently in a disassociated state. This is consistent with the apparent divalent metal-mediated decompaction we observe for offcentered NCP, which is missing an H3 N-terminal tail-DNA interaction at one terminus, and previous work has indicated that the $\mathrm{H} 3$ tail is critical for maintaining nucleosome stability in any case [41]. Accordingly, HMGN2 and HMGN1t apparently act in a synergistic capacity with divalent metal to further decrease stability of the nucleosome termini. Although the combined nucleosome destabilization arises, at least in part, from a direct effect of $\mathrm{Mg}^{2+}$ or $\mathrm{Ca}^{2+}$, divalent metal binding to acidic residues abundant in the HMGN C-termini-potentially altering protein conformation-may also be involved in the activity.

\section{Conclusions}

We observe divalent metal-dependent stability of the nucleosome core and differences in the nucleosome modulation activities of HMGN1 and HMGN2. This emphasizes the potential importance of testing the influence of $\mathrm{Mg}^{2+}$ and $\mathrm{Ca}^{2+}$ in chromatin studies. Within our in vitro system established here, HMGN2 appears capable of destabilizing 
the nucleosome core, while HMGN1 may require additional factors for similar activity. Further investigations, which include extra nuclear components or probe the influence of posttranslational modifications, could help elucidate the mechanism of chromatin unfolding by the HMGNs.

\section{Author Contributions}

M. S. Ong carried out nucleosome array experiments and thermal denaturation assays; D. Vasudevan produced HMGN produced HMGNs and conducted and conducted NCP experiments; C. A. Davey designed research and wrote the manuscript.

\section{Acknowledgments}

This work was supported by the A*STAR Biomedical Research Council, Singapore (grant no. 06/1/22/19/452). The authors thank T. Richmond for providing the nucleosome array constructs. They are grateful to B. Wu, R. Muhammad, J. Yan and H. Fu for critical input on the project and G. E. Davey for comments on the paper. Michelle S. Ong and Dileep Vasudevan, contributed equally in this work.

\section{References}

[1] R. D. Kornberg and Y. Lorch, "Chromatin rules," Nature Structural and Molecular Biology, vol. 14, no. 11, pp. 986-988, 2007.

[2] T. J. Richmond and C. A. Davey, "The structure of DNA in the nucleosome core," Nature, vol. 423, no. 6936, pp. 145-150, 2003.

[3] C. Jiang and B. F. Pugh, "Nucleosome positioning and gene regulation: advances through genomics," Nature Reviews Genetics, vol. 10, no. 3, pp. 161-172, 2009.

[4] F. Thoma, "Repair of UV lesions in nucleosomes-intrinsic properties and remodeling," DNA Repair, vol. 4, no. 8, pp. 855-869, 2005.

[5] S. Henikoff, "Nucleosome destabilization in the epigenetic regulation of gene expression," Nature Reviews Genetics, vol. 9, no. 1, pp. 15-26, 2008.

[6] Y. Postnikov and M. Bustin, "Regulation of chromatin structure and function By HMGN proteins," Biochimica et Biophysica Acta, vol. 1799, no. 1-2, pp. 62-68, 2010.

[7] M. Bustin, "Chromatin unfolding and activation by HMGN* chromosomal protiens," Trends in Biochemical Sciences, vol. 26, no. 7, pp. 431-437, 2001.

[8] M. Subramanian, R. W. Gonzalez, H. Patil et al., "The nucleosome-binding protein HMGN2 modulates global genome repair," The FEBS Journal, vol. 276, no. 22, pp. 66466657, 2009.

[9] T. Ueda, Y. V. Postnikov, and M. Bustin, "Distinct domains in high mobility group $\mathrm{N}$ variants modulate specific chromatin modifications," Journal of Biological Chemistry, vol. 281, no. 15, pp. 10182-10187, 2006.

[10] S. Cherukuri, R. Hock, T. Ueda, F. Catez, M. Rochman, and M. Bustin, "Cell cycle-dependent binding of HMGN proteins to chromatin," Molecular Biology of the Cell, vol. 19, no. 5, pp. 1816-1824, 2008.

[11] M. M. Lucey, Y. Wang, M. Bustin, and M. K. Duncan, "Differential expression of the HMGN family of chromatin proteins during ocular development," Gene Expression Patterns, vol. 8, no. 6, pp. 433-437, 2008.

[12] Y. V. Postnikov, L. Trieschmann, A. Rickers, and M. Bustin, "Homodimers of chromosomal proteins HMG-14 end HMG17 in nucleosome cores," Journal of Molecular Biology, vol. 252, no. 4, pp. 423-432, 1995.

[13] M. P. Crippa, P. J. Alfonso, and M. Bustin, "Nucleosome core binding region of chromosomal protein HMG-17 acts as an independent functional domain," Journal of Molecular Biology, vol. 228, no. 2, pp. 442-449, 1992.

[14] T. Ueda, F. Catez, G. Gerlitz, and M. Bustin, "Delineation of the protein module that anchors HMGN proteins to nucleosomes in the chromatin of living cells," Molecular and Cellular Biology, vol. 28, no. 9, pp. 2872-2883, 2008.

[15] L. Trieschmann, Y. V. Postnikov, A. Rickers, and M. Bustin, "Modular structure of chromosomal proteins HMG-14 and HMG-17: definition of a transcriptional enhancement domain distinct from the nucleosomal binding domain," Molecular and Cellular Biology, vol. 15, no. 12, pp. 6663-6669, 1995.

[16] H.-F. Ding, M. Bustin, and U. Hansen, "Alleviation of historic H1-mediated transcriptional repression and chromatin compaction by the acidic activation region in chromosomal protein HMG-14," Molecular and Cellular Biology, vol. 17, no. 10, pp. 5843-5855, 1997.

[17] J.-H. Lim, F. Catez, Y. Birger, Y. V. Postnikov, and M. Bustin, "Preparation and functional analysis of HMGN proteins," Methods in Enzymology, vol. 375, pp. 323-342, 2004.

[18] K. Luger, T. J. Rechsteiner, and T. J. Richmond, "Preparation of nucleosome core particle from recombinant histones," Methods in Enzymology, vol. 304, pp. 3-19, 1999.

[19] C. A. Davey, D. F. Sargent, K. Luger, A. W. Maeder, and T. J. Richmond, "Solvent mediated interactions in the structure of the nucleosome core particle at $1.9 \AA$ resolution," Journal of Molecular Biology, vol. 319, no. 5, pp. 1097-1113, 2002.

[20] B. Wu and C. A. Davey, "Platinum drug adduct formation in the nucleosome core alters nucleosome mobility but not positioning," Chemistry and Biology, vol. 15, no. 10, pp. 10231028, 2008.

[21] P. T. Lowary and J. Widom, "New DNA sequence rules for high affinity binding to histone octamer and sequence-directed nucleosome positioning," Journal of Molecular Biology, vol. 276, no. 1, pp. 19-42, 1998.

[22] B. Dorigo, T. Schalch, K. Bystricky, and T. J. Richmond, "Chromatin fiber folding: requirement for the histone $\mathrm{H} 4 \mathrm{~N}$ terminal tail," Journal of Molecular Biology, vol. 327, no. 1, pp. 85-96, 2003.

[23] T. Schalch, S. Duda, D. F. Sargent, and T. J. Richmond, "Xray structure of a tetranucleosome and its implications for the chromatin fibre," Nature, vol. 436, no. 7047, pp. 138-141, 2005.

[24] M. S. Ong, T. J. Richmond, and C. A. Davey, "DNA stretching and extreme kinking in the nucleosome core," Journal of Molecular Biology, vol. 368, no. 4, pp. 1067-1074, 2007.

[25] P. N. Bryan, E. B. Wright, and M. H. Hsie, "Physical properties of inner histone-DNA complexes," Nucleic Acids Research, vol. 5, no. 10, pp. 3603-3617, 1978.

[26] W. O. Weischet, K. Tatchell, K. E. Van Holde, and H. Klump, "Thermal denaturation of nucleosomal core particles," Nucleic Acids Research, vol. 5, no. 1, pp. 139-160, 1978.

[27] R. T. Simpson, "Structure of the chromatosome, a chromatin particle containing 160 base pairs of DNA and all the histones," Biochemistry, vol. 17, no. 25, pp. 5524-5531, 1978.

[28] A. E. Paton, E. Wilkinson-Singley, and D. E. Olins, "Nonhistone nuclear high mobility group proteins 14 and 17 stabilize 
nucleosome core particles," Journal of Biological Chemistry, vol. 258, no. 21, pp. 13221-13229, 1983.

[29] M. P. Crippa, L. Trieschmann, P. J. Alfonso, A. P. Wolffe, and M. Bustin, "Deposition of chromosomal protein HMG17 during replication affects the nucleosomal ladder and transcriptional potential of nascent chromatin," The EMBO Journal, vol. 12, no. 10, pp. 3855-3864, 1993.

[30] L. Trieschmann, P. J. Alfonso, M. P. Crippa, A. P. Wolffe, and M. Bustin, "Incorporation of chromosomal proteins HMG14/HMG-17 into nascent nucleosomes induces an extended chromatin conformation and enhances the utilization of active transcription complexes," The EMBO Journal, vol. 14, no. 7, pp. 1478-1489, 1995.

[31] J.-H. Lim, M. Bustin, V. V. Ogryzko, and Y. V. Postnikov, "Metastable macromolecular complexes containing high mobility group nucleosome-binding chromosomal proteins in HeLa nuclei," Journal of Biological Chemistry, vol. 277, no. 23, pp. 20774-20782, 2002.

[32] Y. V. Postnikov, J. E. Herrera, R. Hock, U. Scheer, and M. Bustin, "Clusters of nucleosomes containing chromosomal protein HMG-17 in chromatin," Journal of Molecular Biology, vol. 274, no. 4, pp. 454-465, 1997.

[33] R. Strick, P. L. Strissel, K. Gavrilov, and R. Levi-Setti, "Cationchromatin binding as shown by ion microscopy is essential for the structural integrity of chromosomes," Journal of Cell Biology, vol. 155, no. 6, pp. 899-910, 2001.

[34] D. A. Hill, C. L. Peterson, and A. N. Imbalzano, "Effects of HMGN1 on chromatin structure and SWI/SNF-mediated chromatin remodeling," Journal of Biological Chemistry, vol. 280, no. 50, pp. 41777-41783, 2005.

[35] K. L. West, Y. Ito, Y. Birger, Y. Postnikov, H. Shirakawa, and M. Bustin, "HMGN3a and HMGN3b, two protein isoforms with a tissue-specific expression pattern, expand the cellular repertoire of nucleosome-binding proteins," Journal of Biological Chemistry, vol. 276, no. 28, pp. 25959-25969, 2001.

[36] J. G. Duguid, V. A. Bloomfield, J. M. Benevides, and G. J. Thomas Jr., "Raman spectroscopy of DNA-metal complexes. II. The thermal denaturation of DNA in the presence of $\mathrm{Sr}^{2+}$, $\mathrm{Ba}^{2+}, \mathrm{Mg}^{2+}, \mathrm{Ca}^{2+}, \mathrm{Mn}^{2+}, \mathrm{Co}^{2+}, \mathrm{Ni}^{2+}$, and $\mathrm{Cd}^{2+}, "$ Biophysical Journal, vol. 69, no. 6, pp. 2623-2641, 1995.

[37] B. Wu and C. A. Davey, "Using soft X-Rays for a detailed picture of divalent metal binding in the nucleosome," Journal of Molecular Biology, vol. 398, no. 5, pp. 633-640, 2010.

[38] K. J. Polach and J. Widom, "Mechanism of protein access to specific DNA sequences in chromatin: a dynamic equilibrium model for gene regulation," Journal of Molecular Biology, vol. 254, no. 2, pp. 130-149, 1995.

[39] J. D. Anderson and J. Widom, "Sequence and positiondependence of the equilibrium accessibility of nucleosomal DNA target sites," Journal of Molecular Biology, vol. 296, no. 4, pp. 979-987, 2000.

[40] G. Li, M. Levitus, C. Bustamante, and J. Widom, "Rapid spontaneous accessibility of nucleosomal DNA," Nature Structural and Molecular Biology, vol. 12, no. 1, pp. 46-53, 2005.

[41] H. Ferreira, J. Somers, R. Webster, A. Flaus, and T. OwenHughes, "Histone tails and the $\mathrm{H} 3 \alpha \mathrm{N}$ helix regulate nucleosome mobility and stability," Molecular and Cellular Biology, vol. 27, no. 11, pp. 4037-4048, 2007. 

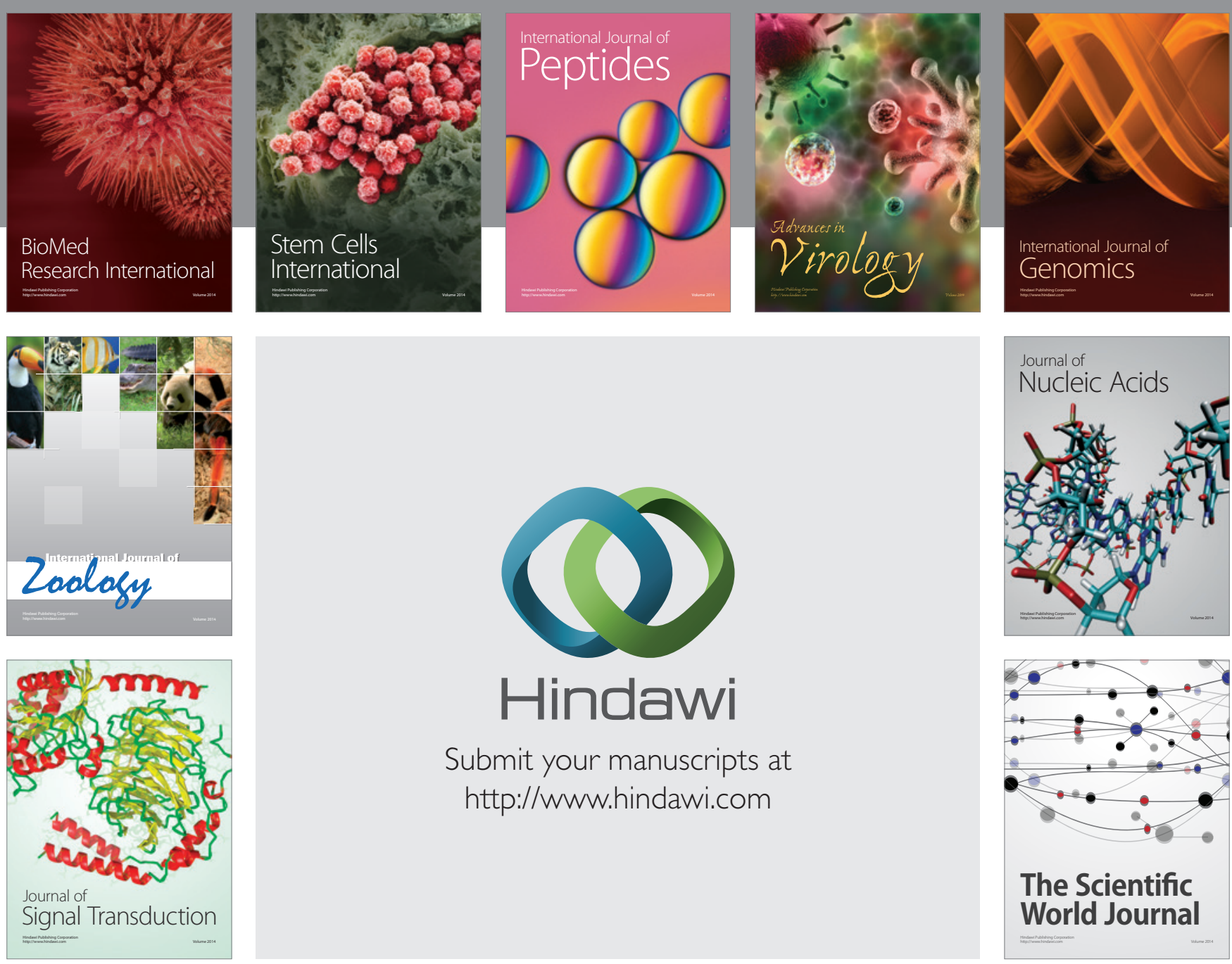

Submit your manuscripts at

http://www.hindawi.com
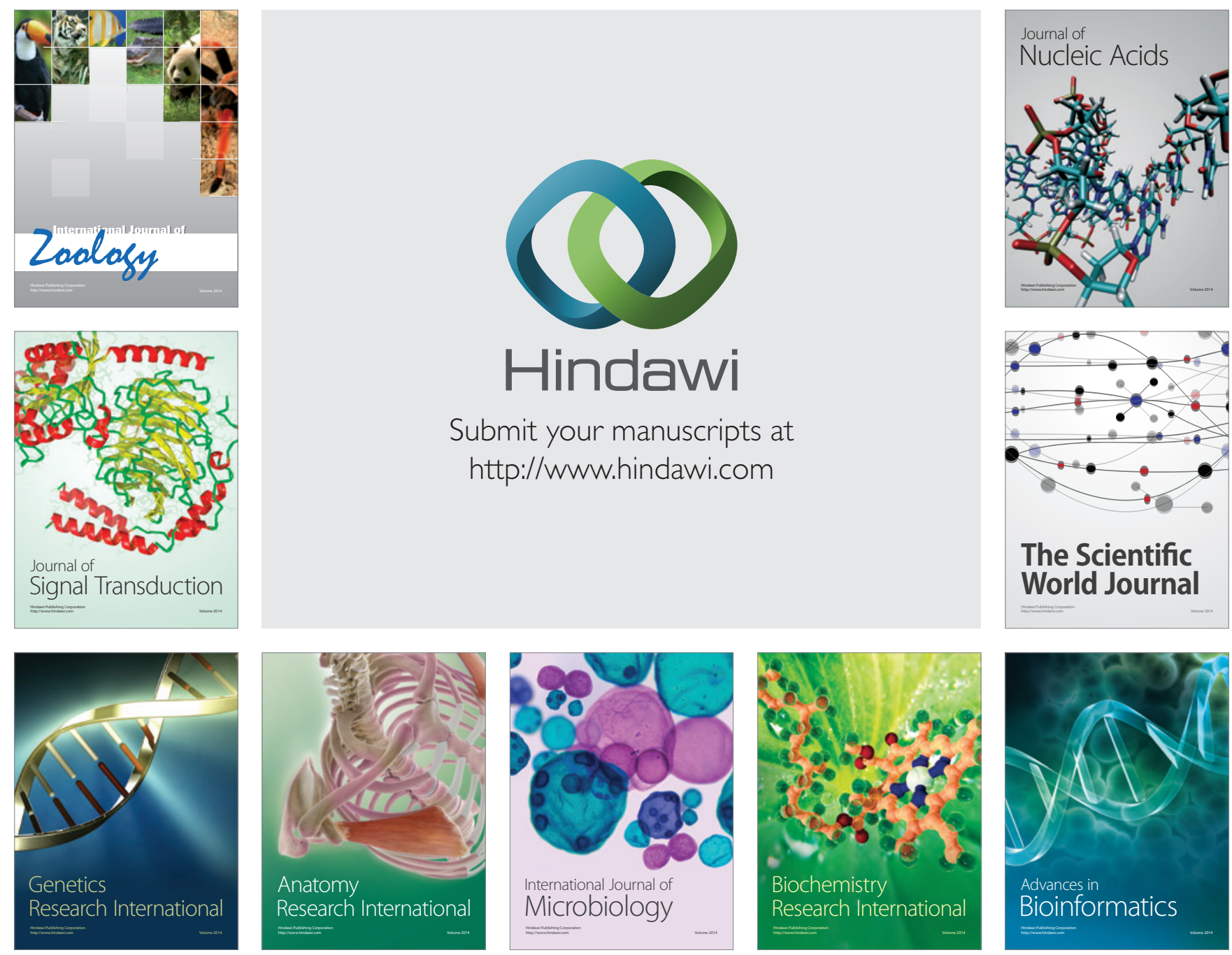

The Scientific World Journal
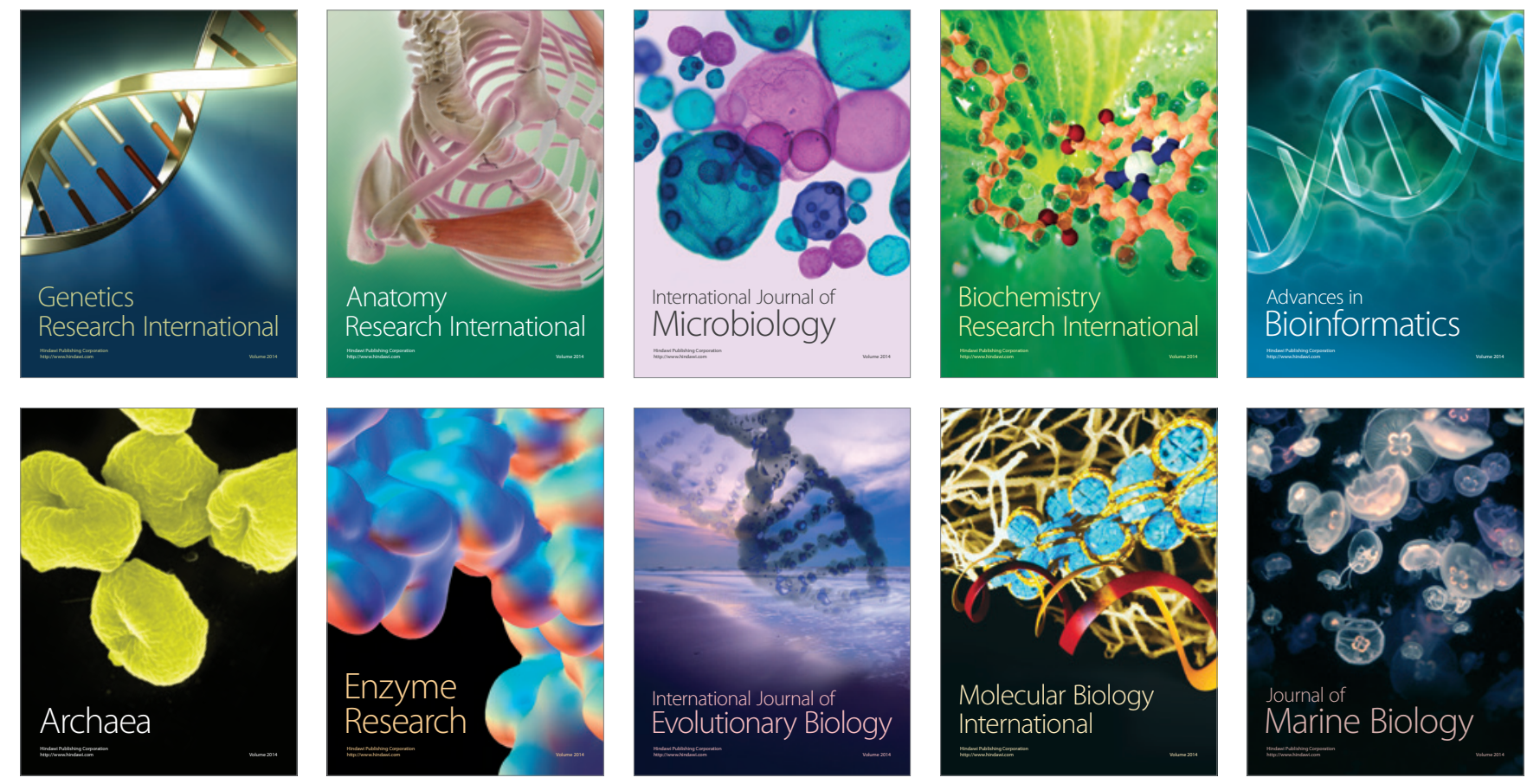